\title{
IMPLEMENTATION OF A TREE CODE FOR COSMOLOGY
}

\author{
François R. Bouchet ${ }^{1,2,3}$ and Lars Hernquist ${ }^{1,2}$ \\ ${ }^{1}$ Department of Astronomy, U.C. Berkeley. \\ ${ }^{2}$ IGPP, Lawrence Livermore National Laboratory. \\ ${ }^{3}$ On leave from Institut d'Astrophysique de Paris.
}

We have adapted the Barnes-Hut hierarchical N-body method for cosmological applications. A detailed study of the resulting code yielded the following conclusions: it is SIMPLE, FLEXIBLE, ACCURATE, ROBUST, and EFFICIENT.

-SIMPLE: in the hierarchical method the force exerted on a particle by a distant cluster of particles is evaluated using a truncated multipole expansion. In our implementation, only the monopole term is retained. Consider the ratio of the transverse size of a cluster, $s$, to its distance from a particle, $d$. If $s / d$ is greater than some fixed value $\theta$, the cluster is divided into subcomponents. This procedure can be performed efficiently if the particles are organized into a nested hierarchy of clusters or cells using a tree data structure. Our code is based on a recursive division of space into cubic cells. The spatial resolution is not limited by a grid. -FLEXIBLE: apart from the easy accuracy control through the value given to $\theta$, it is also possible to consider various geometries and boundary conditions, i.e. freelyexpanding, quasi-, and fully-periodic boundaries. (In the quasi-periodic case, the forces are computed as if the box was always centered on the particles, but there are no replicas of the distribution outside of the centered box.)

-ACCURATE: a simple way to estimate the accuracy of the code is to compare the results with those of an $N^{2}$ code, in which the forces are computed exactly by summing all the 2-body interactions. We performed detailed comparisons of the evolution of 4096 particles. Visual inspection, computation of 2-point correlation functions, and measurement of the relative differences in the computed forces showed very good agreement between the 2 sets of experiments. We have also tested the code for $N$ up to $32768, a \leq 30$ (where $a$ is the expansion factor), and a smoothing parameter down to $\lambda / 50$, where $\lambda$ is the mean interparticle separation. In all cases, the energy conservation violation was $\Delta E / \Delta(a U) \sim f(a \theta) / \theta \leq 0.2 \%$. -ROBUST: for sufficiently small values of $\theta(\simeq 0.75)$, the variations in the dynamical evolution due to the errors in the force computation are negligible compared with variations resulting from statistical fluctuations in the initial conditions.

-EFFICIENT: the computation time scales with the particle number $N$ as $N \log N$ (as a Fourier code), and with $\theta$ as $\theta^{-2}$. Finally, and most importantly, the cpu time per step is nearly independent of the level of clustering, unlike a $\mathrm{P}^{3} \mathrm{M}$ code.

This method will thus allow simulations with many particles $(N \sim 100,000)$, and with a high spatial resolution, deep into the non-linear regime. 